\title{
Magnetic field sustained by the elastic force in neutron star crusts
}

\author{
Yasufumi Kojima $^{\star 1}$, Shota Kisaka ${ }^{1}$, Kotaro Fujisawa ${ }^{2}$ \\ ${ }^{1}$ Department of Physics, Graduate School of Advanced Science and Engineering, Hiroshima University, \\ Higashi-Hiroshima, Hiroshima 739-8526, Japan \\ ${ }^{2}$ Research Center for Early Universe, Graduate School of Science, University of Tokyo, Bunkyo-ku, Tokyo 113-0033, Japan
}

7 January 2022

\begin{abstract}
We investigate the magneto-elastic equilibrium of a neutron star crust and magnetic energy stored by the elastic force. The solenoidal motion driven by the Lorentz force can be controlled by the magnetic elastic force, so that conditions for the magnetic field strength and geometry are less restrictive. For equilibrium models, the minor solenoidal part of the magnetic force is balanced by a weak elastic force because the irrotational part is balanced by the dominant gravity and pressure forces. Therefore, a strong magnetic field may be confined in the interior, regardless of poloidal or toroidal components. We numerically calculated axially symmetric models with the maximum shear-strain, and found that a magnetic energy $>10^{46}$ erg can be stored in the crust, even for a normal surface dipole-field-strength $\left(<10^{13}\right.$ $\mathrm{G})$. The magnetic energy much exceeds the elastic energy $\left(10^{44}-10^{45} \mathrm{erg}\right)$. The shear-stress spatial distribution revealed that the elastic structure is likely to break down near the surface. In particular, the critical position is highly localized at a depth less than $100 \mathrm{~m}$ from the surface.
\end{abstract}

Key words: stars: neutron stars: magnetars: magnetic fields

\section{INTRODUCTION}

Neutron stars are generally observed as radio pulsars, and their magnetic field strength is estimated to be $\sim 10^{12} \mathrm{G}$ using the spin period and its derivative. However, some isolated neutron stars exhibit a field strength higher than $10^{14}$ G. Such neutron stars do not typically exhibit radio emissions, but emit occasional energetic bursts or anomalous X-ray luminosities. The rotation rate for such pulsars cannot account for the anomalous emissions(see Turolla et al. 2015; Enoto et al. 2019, for reviews). The output energy for such objects can be attributed to strong magnetic fields, and these objects are called magnetars. However, the surface dipole strength cannot be used to distinguish magnetars and pulsars. There are some exceptions such as low-field magnetars $B=6 \times 10^{12}-4 \times 10^{13} \mathrm{G}$ (Rea et al. 2012, 2013, 2014), and high-magnetic field radio pulsars, such as $B=9.4 \times 10^{13} \mathrm{G}$ in PSR J1847-0130 (McLaughlin et al. 2003). Moreover, magnetar-like X-ray outburst from a high-magnetic field radio pulsar PSR J1119-6127 with $B=4.1 \times 10^{13} \mathrm{G}$ has been reported previously (Archibald et al. 2016). The boundary between magnetars and high-magnetic field radio pulsars has not been elucidated.

The surface dipole field of young neutron stars in some supernova remnants, called central compact objects (CCOs) (Gotthelf et al. 2013; De Luca 2017), has been estimated to be $\sim 10^{11}$ G. Furthermore, their X-ray luminosities exceed the kinetic energy losses, and are comparable to those of quiescent magnetars (Kaspi \& Beloborodov 2017). Therefore, a considerable magnetic energy of $\sim 10^{47} \mathrm{erg}$ is stored in such objects, and the field strength is $\sim 10^{15} \mathrm{G}$ in the stellar interior. Recently, the magneto-thermal evolution of strong and highly tangled magnetic fields in neutron star crusts has been calculated to account for the CCO power (Gourgouliatos et al. 2020; Igoshev et al. 2021; De Grandis et al. 2021).

In some neutron stars, a strong magnetic field $>10^{14} \mathrm{G}$ is crucial for various phenomena. However, the locations and configurations of such strong fields have not been clarified, and a theoretical understanding of such fields is desired. Although the stability of magnetized stars has attracted considerable research interest, the underlying phenomenon has not yet been clarified. Simple configurations such as purely toroidal and poloidal magnetic field components are unstable (Tayler 1973; Markey \& Tayler 1973; Wright 1973), and a twisted-torus configuration is considered a stable equilibrium configuration, in which both components (toroidal and poloidal) stabilize each other.

The stability of magnetic stars has been examined numerically (e.g., Braithwaite \& Nordlund 2006; Lander \& Jones 2011a,b; Mitchell et al. 2015), following the pioneering work in Braithwaite \& Spruit (2004). Dynamical simulations revealed that the final state after a few Alfvén-wave crossing times is a twisted-torus configuration, in which the poloidal and toroidal components

* E-mail: ykojima-phys@hiroshima-u.ac.jp

(C) 0000 The Authors 
of comparable field strengths tangle with each other. The fraction of the toroidal component to the total magnetic energy is greater than $\sim 0.2$ (Braithwaite 2009; Duez et al. 2010; Sur et al. 2020).

Static or stationary axially symmetric equilibrium models have been calculated for various conditions using several methods (e.g. Tomimura \& Eriguchi 2005; Yoshida \& Eriguchi 2006; Yoshida et al. 2006; Lander \& Jones 2009; Ciolfi et al. 2009; Duez \& Mathis 2010; Fujisawa \& Eriguchi 2013; Gourgouliatos et al. 2013; Armaza et al. 2015), and such analyses have been extended to relativistic models (e.g. Uryū et al. 2019, and references therein). However, the results reveal that the toroidal magnetic energy in equilibrium is not high. Instead, special conditions such as magnetic fields completely confined in deep stellar interiors (Duez \& Mathis 2010), or strong surface currents (Colaiuda et al. 2008; Fujisawa \& Eriguchi 2013; Fujisawa \& Kisaka 2014) may be necessary to construct models with a large toroidal field. In addition to the mixed field configuration, the stratified structure of the density and pressure is a critical factor for stabilizing such systems (e.g., Glampedakis et al. 2012; Akgün et al. 2013; Yoshida 2019), as reported in previous studies (Tayler 1980).

Elastic stress, which is another factor in a realistic neutron star model, has been investigated using dynamic (Bera et al. 2020) and static calculations (Kojima et al. 2021), and the results show that elasticity is crucial for stability. In a previous study (Kojima et al. 2021), we considered the static equilibrium of a magnetized crust with elastic force, and demonstrated that the crust sustained considerable magnetic energy $\left(>10^{46} \mathrm{erg}\right.$ ). However, we approximated the shear modulus as a constant throughout the crust. In this paper, we study the magneto-elastic equilibrium in more detail using a realistic shear modulus that changes by a factor of $10^{3}$ in the neutron-star crust. Moreover, we clarify the spatial dependence of the elastic force and determine the point where the elastic limit breaks down. Our results will be of astrophysical relevance in studying abrupt bursts and magnetic field evolution in a secular timescale.

The remainder of this manuscript is organized as follows. Section 2 presents the models and equations relevant to our problem. The shear modulus is assumed to be proportional to density, and the elastic force is formulated. The numerical results are presented in Section 3. Finally, Section 4 concludes this paper.

\section{MATHEMATICAL FORMULATION}

\subsection{Force balance}

We consider an equilibrium state for the crust of a magnetized neutron star and use spherical coordinates $(r, \theta, \varphi)$ to represent an axially symmetric configuration $\partial_{\varphi}=0$. The static force balance for a nonrotating star in the Newtonian gravity is given by $-\vec{\nabla} P-\rho \vec{\nabla} \Phi_{\mathrm{G}}+\vec{f}+\vec{h}=0$

where the first two terms expressed by pressure $P$, mass density $\rho$, and gravitational potential $\Phi_{\mathrm{G}}$ are dominant. We further assume a barotropic distribution, $P=P(\rho)$, and the sum of these forces is expressed by $-\rho \vec{\nabla} \Phi_{\text {eff }}$. The resultant stellar structure is spherically symmetric. The barotropic approximation restricts the configuration such that no stable equilibrium exists (e.g. Reisenegger 2009; Lander \& Jones 2012). However, we adopt the barotropic approximation to examine the effect of the elastic force in a simplified setup. Otherwise, a solenoidal acceleration $\vec{\nabla} \times\left(\rho^{-1} \nabla P\right) \neq 0$ is generated in a non-barotropic star.

The third term in eq.(1) is the Lorentz force $\vec{f} \equiv c^{-1} \vec{j} \times \vec{B}$, which is considerably smaller than the dominant forces and different in nature. Typically, the Lorentz force causes non-radial acceleration. The static force-balance with the Lorentz force constrains the field strength and configuration of the magnetic field. For example, the energy ratio of the toroidal to poloidal components is limited to small values for barotropic magnetohydrodynamic (MHD) equilibrium, as discussed in Section 1.

This constraint can be relaxed considerably by incorporating the elastic force $\vec{h}$, irrespective of its small magnitude (Kojima et al. 2021). The Lorentz force comprises irrotational and solenoidal parts. The irrotational force is easily balanced by the dominant forces, but the solenoidal force is never balanced. However, the elastic force can be used to balance the solenoidal component of the Lorentz force.

Equation(1) cannot be directly solved for magneto-elastic equilibrium models, because the order of their strengths differs considerably; the Lorentz $\vec{f}$ and elastic $\vec{h}$ forces are considerably smaller than the gravity and pressure forces. An approximation is given by

$\vec{f}+\vec{h}=0$.

The weak forces are balanced under fixed stellar structures. Instead of eq.(2), we use a weaker constraint. Thus, in the solenoidal part, the "curl" of eq.(1) should be balanced between $\vec{f}$ and $\vec{h}$, because the irrotational part may be balanced by a small perturbation in the pressure and gravity. A set of approximated equations is as follows:

$(\vec{f}+\vec{h})_{\varphi}=0$

$\left[\vec{\nabla} \times \rho^{-1}(\vec{f}+\vec{h})\right]_{\varphi}=0$.

We consider the azimuthal component only in eq.(4), because the poloidal components vanish, as evident from eq.(3) and the axial symmetry $\left(\partial_{\varphi}=0\right)$. 


\subsection{Elastic force in the crust}

Our consideration of the magnetic-elastic equilibrium in the solid crust of a neutron star is limited to the inner crust, where the mass density $\rho$ ranges from $\rho_{c}=1.4 \times 10^{14} \mathrm{~g} \mathrm{~cm}^{-3}$ at the core-crust boundary $r_{c}$ to the neutron-drip density $\rho_{1}=4 \times 10^{11} \mathrm{~g}$ $\mathrm{cm}^{-3}$ at $R$. We ignore the outer crust, which could be considerably affected in the presence of a strong magnetic field, and assume the exterior region of $r>R$ as the vacuum. The spatial profile for $r_{c} \leq r \leq R$ is approximated as (Lander \& Gourgouliatos 2019; Kojima \& Suzuki 2020).

$\frac{\rho}{\rho_{c}}=\left[1-\left(1-\left(\frac{\rho_{1}}{\rho_{c}}\right)^{1 / 2}\right)\left(\frac{r-r_{c}}{d}\right)\right]^{2}$,

where $d=R-r_{c}$ is the crust thickness, assumed to be $d / R=0.1$.

The shear modulus $\mu$ increases with the density, and may be approximated as a linear function of $\rho$, which is overall fitted to the results of a detailed calculation reported previously (see Fig. 43 in Chamel \& Haensel 2008). $\mu$ is expressed as $\mu=\frac{\mu_{1} \rho}{\rho_{1}}$

where $\mu_{1}=3 \times 10^{27} \mathrm{erg} \mathrm{cm}^{-3}$ at the outer boundary. The value increases with $\rho$ until $\mu_{c} \approx 10^{30} \mathrm{erg} \mathrm{cm}^{-3}$ at the core- crust interface.

The elastic force $\vec{h}$ in the solid crust is considered, and the $i$ th component $h_{i}$ is expressed using the trace-free strain tensor $\sigma_{i j}$ and $\mu$ as

$h_{i}=\nabla_{j}\left(\mu \sigma_{i}^{j}\right)$

where $\sigma_{i j}$ is expressed in terms of the displacement vector $\xi_{i}$ and three-dimensional metric $g_{i j}$ as

$\sigma_{i j}=\nabla_{i} \xi_{j}+\nabla_{j} \xi_{i}-\frac{2}{3} g_{i j}\left(\nabla_{k} \xi^{k}\right)$

The components of eq.(7) in spherical coordinates is expressed as

$$
\begin{aligned}
h_{r} & =\left[-\vec{\nabla} \times\left(\mu \vec{\omega}_{\varphi}\right)+2 \vec{\nabla}(\mu \vec{\nabla} \cdot \vec{\xi})\right]_{r}-2 \mu_{, r}\left[\frac{2}{r} \xi_{r}+\frac{1}{\varpi}\left(\sin \theta \xi_{\theta}\right)_{, \theta}\right]+\frac{2 \mu_{, \theta}}{r} \xi_{\theta, r}, \\
h_{\theta} & =\left[-\vec{\nabla} \times\left(\mu \vec{\omega}_{\varphi}\right)+2 \vec{\nabla}(\mu \vec{\nabla} \cdot \vec{\xi})\right]_{\theta}+\frac{2 \mu_{, r}}{r}\left(\xi_{r, \theta}-\xi_{\theta}\right)-\frac{2 \mu, \theta}{r \varpi}\left[\cos \theta \xi_{\theta}+\sin \theta\left(r \xi_{r}\right)_{, r}\right], \\
h_{\varphi} & =\frac{1}{\varpi^{3}}\left[\mu \varpi^{4}\left(\frac{\xi_{\varphi}}{\varpi}\right)_{, r}\right]_{, r}+\frac{1}{r^{2} \varpi^{2}}\left[\mu \varpi^{3}\left(\frac{\xi_{\varphi}}{\varpi}\right)_{, \theta}\right]_{, \theta},
\end{aligned}
$$

where

$\vec{\omega}_{\varphi}=\vec{\nabla} \times \vec{\xi}_{p}=\frac{W}{\varpi} \vec{e}_{\varphi}$,

and $\varpi=r \sin \theta$ is the cylindrical radius; $\vec{e}_{\varphi}$ is the unit vector along the azimuthal direction. In eq.(12), the azimuthal vector $\vec{\omega}_{\varphi}$ is expressed as a scalar function $W$.

We limit our consideration to incompressible motion and introduce a function $X$ to express $\vec{\xi}_{p}$ as given below, which satisfies the condition $\vec{\nabla} \cdot \vec{\xi}=0$,

$\vec{\xi}_{p}=\vec{\nabla} \times\left(\frac{X}{\varpi} \vec{e}_{\varphi}\right)$

Equation (12) is reduced to a relation between $X$ and $W$ as

$\left[\vec{\nabla} \times \vec{\nabla} \times\left(\frac{X}{\varpi} \vec{e}_{\varphi}\right)\right]_{\varphi}=\frac{W}{\varpi}$.

Under the approximation $\mu=\mu(r)$, the elastic acceleration term in eq.(4) with eqs.(9) and (10) is expressed by

$$
\left(\vec{\nabla} \times \rho^{-1} \vec{h}\right)_{\varphi}=-\left[\vec{\nabla} \times\left(\rho^{-1} \vec{\nabla} \times\left(\frac{\mu W}{\varpi} \vec{e}_{\varphi}\right)\right)\right]_{\varphi}+\frac{2}{\varpi}\left[\left(\frac{\rho^{-1} \mu^{\prime}}{r} X_{, r}\right)_{, r}+\frac{\left(\rho^{-1} \mu^{\prime}\right)^{\prime} \sin \theta}{r^{2}}\left(\frac{1}{\sin \theta} X_{, \theta}\right)_{, \theta}\right],
$$

where a prime' denotes derivative with respect to $r$.

\subsection{Magnetic field}

An axially symmetric magnetic field is described using two functions as

$\vec{B}=\vec{\nabla} \times\left(\frac{\Psi}{\varpi} \vec{e}_{\varphi}\right)+\frac{S}{\varpi} \vec{e}_{\varphi}$,

and the electric currents are derived from $\vec{B}$ using the Ampére-Biot-Savart law

$\frac{4 \pi}{c} \vec{j}=\vec{\nabla} \times\left(\frac{S}{\varpi} \vec{e}_{\varphi}\right)+\vec{\nabla} \times \vec{\nabla} \times\left(\frac{\Psi}{\varpi} \vec{e}_{\varphi}\right)$. 


\begin{tabular}{cll} 
Name & Magnetic field geometry & Elastic motion \\
\hline $\mathrm{P}$ & Confined poloidal field expressed by eq.(20) & $\vec{\xi}_{p}$ \\
$\mathrm{~T}$ & Confined toroidal field expressed by eq.(21) & $\vec{\xi}_{p}, \xi_{\varphi}$ \\
$\mathrm{M}$ & Mixed poloidal-toroidal field using eqs.(20) and $(21)$, & $\overrightarrow{\xi_{p}}, \xi_{\varphi}$ \\
\hline
\end{tabular}

Table 1. Description of the magnetic field for the equilibrium model with elastic force

The current function $S$ is a function of $\Psi$, when the azimuthal component of the Lorentz force vanishes, $f_{\varphi}=0$. An irrotational condition $\vec{\nabla} \times\left(\rho^{-1} \vec{f}\right)=0$ constrains the azimuthal current as

$\frac{4 \pi \varpi}{c} j_{\varphi}=-\rho K^{\prime} \varpi^{2}+S^{\prime} S$

where $K$ is a function of $\Psi$, and a prime' denotes the derivative with respect to $\Psi$. Thus, the Lorentz force is reduced to

$\vec{f}=-\frac{\rho K^{\prime}}{4 \pi} \vec{\nabla} \Psi$.

A simple model of barotropic MHD equilibrium is obtained by assuming that the field is purely dipolar ( $\Psi \propto \sin ^{2} \theta$ and $S=0$ ), and that $K^{\prime}$ is a constant. We assume that the magnetic field exists outside the core, and the field inside the crust is smoothly connected to the external dipole in vacuum. For the magnetic field in barotropic MHD equilibrium, a solenoidal force does not exist, i.e., the elastic force vanishes, $\vec{\xi}=0$. We adopt this magnetic field configuration as a reference model and examine the response of the elastic force $(\vec{\xi} \neq 0)$ by adding another arbitrary field. We consider the possibility of a strong field confined inside the crust, irrespective of whether the component is poloidal or toroidal. The choice of the models is described as follows, and is listed in Table 1.

The poloidal magnetic field confined in the crust $\left(r_{c} \leq r \leq R\right)$ may be modeled as

$\Psi_{(1)}=N_{1} B_{0} R^{-2}\left[\left(r-r_{c}\right)(r-R)\right]^{2} \sin \theta P_{l}^{\prime}$,

where $P_{l}(\cos \theta)$ is the Legendre polynomial with multipole index $l$, and a prime' denotes the derivative with respect to $\cos \theta$. Similarly, the confined toroidal field $B_{\varphi}=S / \varpi$ may be modeled as

$S_{(2)}=N_{2} B_{0} R^{-3}\left[\left(r-r_{c}\right)(r-R)\right]^{2} \sin \theta P_{l}^{\prime}$.

In eqs. (20) and (21), we use $B_{0} \equiv\left|B_{0}(R, 0)\right|$ for the reference field $\vec{B}_{0}$ at the pole as the normalization of the magnetic field strength; $N_{s}(s=1,2)$ is a dimensionless number. Here, the radial function is selected such that it vanishes at the core-crust interface and at the surface. The electric current $\vec{j}_{(\mathrm{s})}(s=1,2)$ is calculated from $\Psi_{(1)}$ or $S_{(2)}$ using eq.(17).

Three equilibrium sequences are considered, namely models P, T, and M. Model P represents a purely poloidal magnetic field, for which the elastic motion $\vec{\xi}_{p}$ is solely driven by the Lorentz force. The magnitude $\left|\xi_{p}\right|$ increases with $\left|N_{1}\right|$. Model T represents a purely toroidal magnetic field, for which both components $\vec{\xi}_{p}$ and $\xi_{\varphi}$ are driven by the Lorentz force. The magnitude $|\xi|$ also increases with $\left|N_{2}\right|$. Model M represents a mixed model of poloidal and toroidal magnetic fields. The magnitude $|\xi|$ depends on $N_{1}$ and $N_{2}$, and the ratio $\left|N_{2} / N_{1}\right|$ determines the dominant component. We fix the ratio as $\left|N_{2} / N_{1}\right|=10^{2}$, for which the typical magnetic field strength is given by $\left|B_{\varphi}\right| /\left|B_{\mathrm{p}}\right| \sim 10$.

A critical limit of normalization is determined by either $N_{1}$ or $N_{2}$, beyond which the crust does not respond elastically. We discuss the explicit condition in the next subsection. In Section 3, we show the numeric calculation of a state with the maximum strain, characterized by a quantity $\sigma_{c}$. These models depend on the multipole index $l$ for the additional magnetic field and are summarized in Table 1.

\subsection{Elastic limit and parameter}

No elastic shear-deformation $\vec{\xi}=0$ exists in the reference configuration, where the magnetic field and current are denoted as $\vec{B}_{(0)}$ and $\vec{j}_{(0)}$, respectively. We calculate $\vec{\xi}$, which responds to the Lorentz force $\vec{f}=c^{-1} \vec{j} \times \vec{B}$ for $\vec{j}=\vec{j}(0)+\vec{j}_{(s)}$ and $\vec{B}=\vec{B}_{(0)}+\vec{B}_{(s)},(s=1,2)$. The displacement $|\xi|$ in the equilibrium model increases with the normalization constant $\left|N_{\mathrm{s}}\right|$ in eqs.(20) and (21). We adopt the von Mises criterion(e.g., Malvern 1969; Ushomirsky et al. 2000) to estimate the maximum value of $|\xi|$, or the shear tensor $\left|\sigma_{i j}\right|$. The numerical calculation adopting the von Mises criterion is described in the next subsection. The criterion is empirical formula based on the energy. Another criteria, the Tresca, is based on the difference between the maximum and minimum of shear. Two criteria do not predict the same critical states (e.g., Malvern 1969). Our concern is the order-of-magnitude level. The Mises criterion is expressed as follows:

$\frac{1}{2} \sigma_{i j} \sigma^{i j} \leq\left(\sigma_{c}\right)^{2}$

where $\sigma_{c}$ is the maximum strain with a definite value, $\sigma_{c} \approx 10^{-2}-10^{-1}$ (Horowitz \& Kadau 2009; Caplan et al. 2018; Baiko \& Chugunov 2018). 
The elastic limit (22) thus constrains the normalization constant $N_{\mathrm{s}}$. We focus on the equilibrium model with the maximum strain $\sigma_{c}$. The calculated model depends on the ratio $q$ of the elastic and Lorentz forces.

$q \equiv \frac{4 \pi \mu_{1} \sigma_{c}}{B_{0}^{2}} \approx 0.4\left(\frac{B_{0}}{10^{14} \mathrm{G}}\right)^{-2}\left(\frac{\sigma_{c}}{0.1}\right)$.

Here, $B_{0}$ is the magnetic field strength at the pole; the shear modulus, $\mu_{1}=3 \times 10^{27} \mathrm{erg} \mathrm{cm}^{-3}$, at the surface is used. The limit $q=0$ corresponds to our reference model.

The elastic energy stored inside a crust of volume $V$ is given by

$E_{\text {ela }}=\int_{V} \frac{\mu}{2} \sigma_{i j} \sigma^{i j} d V$.

For numerical integration, we fix the stellar model with radius $R=12 \mathrm{~km}$ and crust thickness $d=0.1 R$, and the lowest shear modulus $\mu_{1}=3 \times 10^{27} \mathrm{erg}^{-3}$. The magnetic energy $E_{\mathrm{mag}}$ is given by

$E_{\mathrm{mag}}=\int_{V} \frac{1}{8 \pi} B^{2} d V$

The elastic energy is scaled to $E_{\text {ela }} \propto \sigma_{c}^{2}$ for obtaining an equilibrium model with the maximum strain, whereas the magnetic energy is scaled to $E_{\text {mag }} \propto B_{0}^{2}$. The ratio $E_{\text {ela }} / E_{\text {mag }} \propto \sigma_{c}^{2} B_{0}^{-2}$ with respect to the energy is not equivalent to that of their forces, i.e., $q \propto \sigma_{c} B_{0}^{-2}$.

\subsection{Numerical method}

We numerically calculate the elastic displacement $\vec{\xi}$ against the Lorentz force in the crustal region $r_{c} \leq r \leq R$ with thickness $d / R=\left(R-r_{c}\right) / R=0.1$. In general, the Lorentz force for $\vec{B}_{(0)}+\vec{B}_{(\mathrm{s})}$ and $\vec{j}_{(0)}+\vec{j}_{(\mathrm{s})}$ results in nonzero source terms in eqs.(3) and (4), respectively. We examine the elastic response by solving a set of partial differential equations (3) and (4) with eqs.(11), (14) and (15). To solve these equations, we use expansion via Legendre polynomials $P_{l}(\cos (\theta))$ and radial functions $x_{l}(r), w_{l}(r)$, and $k_{l}(r)$ as

$$
\begin{aligned}
X & =-\sum_{l \geq 1} x_{l} \sin \theta P_{l, \theta}, \\
\mu W & =-\sum_{l \geq 1} w_{l} \sin \theta P_{l, \theta}, \\
\xi_{\varphi} & =-\sum_{l \geq 1} r k_{l} P_{l, \theta} .
\end{aligned}
$$

The source terms derived from the Lorentz force are expanded with radial functions $a_{l}(r)$ and $b_{l}(r)$ as

$f_{\varphi}=-\sum_{l \geq 1} r^{-3} a_{l} P_{l, \theta}$.

$\left[\vec{\nabla} \times \rho^{-1} \vec{f}\right]_{\varphi}=-\sum_{l \geq 1} r^{-1} b_{l} P_{l, \theta}$

By using eqs.(11), (28), and (29), eq.(3) for the azimuthal component, $\xi_{\varphi}$, is deduced to an ordinary differential equation for $k_{l}(l=1,2, \cdots)$

$\left(\mu r^{4} k_{l}^{\prime}\right)^{\prime}-(l-1)(l+2) \mu r^{2} k_{l}=-a_{l}$,

where the orthogonality of the Legendre polynomials is used. Similarly, eq. (14) for the functions $X$ and $W$ becomes

$x_{l}^{\prime \prime}-\frac{l(l+1)}{r^{2}} x_{l}+\frac{1}{\mu} w_{l}=0$.

Equation (4) with eqs.(15) and (30) is also deduced to

$\left(\rho^{-1} w_{l}^{\prime}\right)^{\prime}-\left(\frac{2 \mu^{\prime}}{\mu r}+\frac{l(l+1)}{r^{2}}\right) \rho^{-1} w_{l}+2\left(\frac{\mu^{\prime}}{\rho r}\right)^{\prime}\left(x_{l}^{\prime}-\frac{l(l+1)}{r} x_{l}\right)=-b_{l}$.

In deriving eq.(33), $x_{l}^{\prime \prime}$ is originally used in eq.(15), but is eliminated by eq.(32). The displacement $\vec{\xi}$ is decoupled with respect to the index $l$ because of the spherical symmetry, i.e., $\rho(r)$ and $\mu(r)$. The analytic expressions for $a_{l}(r)$ and $b_{l}(r)$ in the source terms are complicated. We numerically evaluate the coefficients $a_{l}(r)$ and $b_{l}(r)$ in eqs.(29) and (30) for the Lorentz force originated from $\vec{B}_{(0)}+\vec{B}_{(\mathrm{s})}$ and $\vec{j}_{(0)}+\vec{j}_{(\mathrm{s})}$ by using the orthogonality of the Legendre polynomials. In the numerical calculation, we truncated the number of $l$, and determined that the maximum value of $l$ in the range of 40-60 is sufficient for numerical convergence, because the source terms are specified by low multipole $l$ in eqs.(20) and (21).

We discuss the boundary conditions for these radial functions $k_{l}, x_{l}$, and $w_{l}$. The boundary conditions for the axial component $\xi_{\varphi}$ are $\xi_{\varphi}=0$ at $r=r_{c}$ and $\sigma_{r \varphi}=0$, which is $r\left(\xi_{\varphi} / r\right)_{, r}=0$ at $r=R$. Therefore, we impose $k_{l}=0$ at $r_{c}$ and $k_{l}^{\prime}=0$ at $R$. The 

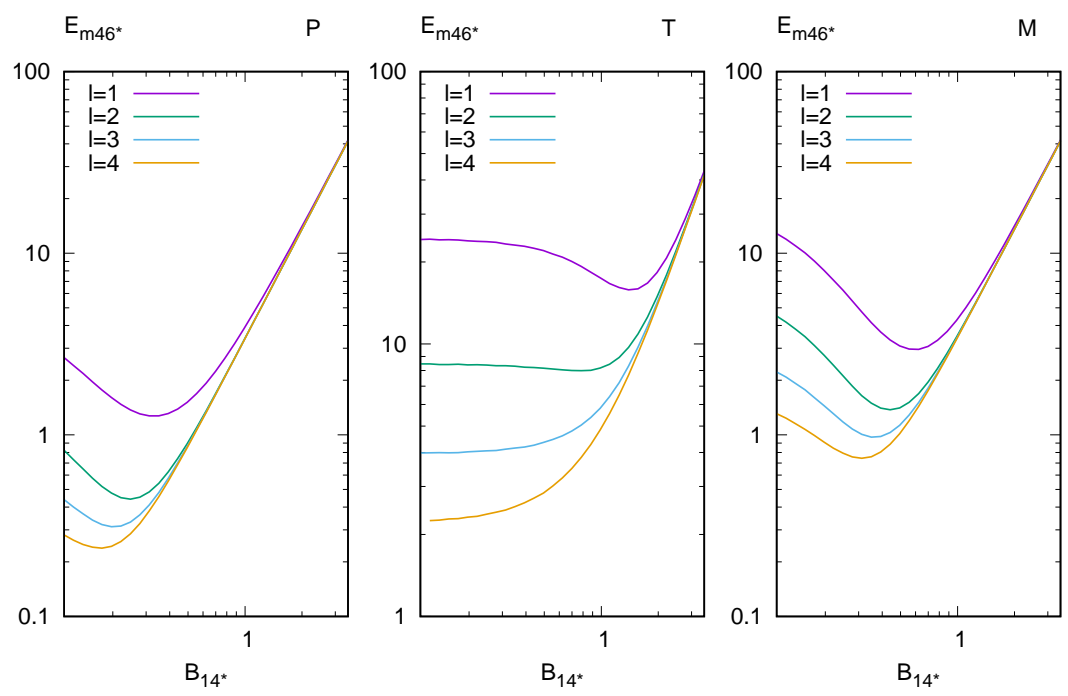

Figure 1. Magnetic energy as a function of the surface dipole field strength for the three models listed in Table 1 for various multipole $l$ values.

direction of the poloidal displacement is horizontal at the radial boundaries. Thus, $x_{l}=0$ at both $r_{c}$ and $R$. Finally, because the shear modulus does not exist outside the crust, $\mu W$ approaches zero at the radial boundaries. Therefore, we impose $w_{l}=0$ at both $r_{c}$ and $R$.

By increasing the normalization constant $\left|N_{\mathrm{s}}\right|$ in eqs.(20) and (21), the source terms $a_{l}$ and $b_{l}$ in eqs.(31) and (33) also increase in magnitude. The maximum displacement $|\xi|$ or the maximum shear-tensor $\left|\sigma_{i j}\right|$ determined by the criterion (22) limits $\left|N_{\mathrm{s}}\right|$. The critical value $\left|N_{\mathrm{s}}\right|$ is determined by numerical solutions of eqs.(31), (32), and (33). The value is relevant to the maximum energy in the elastic limit, whereas it does not affect the spatial distribution of the shear $\sigma_{i j}$, which depends on the magnetic field configuration.

\section{RESULTS}

\subsection{Energy}

The magneto-elastic equilibrium with maximum strain is specified using the dimensionless parameter $q$ in eq.(23), which is the ratio of the elastic and magnetic forces. We calculate various equilibrium models in the range of $q\left(10^{-2.5} \leq q \leq 10^{1.5}\right)$. We first discuss how the magnetic energy (25) depends on the surface dipole field strength. The energy $E_{\text {mag }}$ depends on $B_{0}^{2}$, but we eliminate $B_{0}^{2}$ by $q$, so that $E_{\text {mag }}$ is expressed by $E_{\mathrm{m} 46 *}$ in the ordinate of Fig. 1, where

$E_{\mathrm{mag}} \equiv E_{\mathrm{m} 46 *}\left(\sigma_{c} / 0.1\right) \times 10^{46} \mathrm{erg}$.

Similarly, the abscissa of Fig. 1 is $B_{14 *}$, which is related to $B_{0}$ as

$B_{0} \equiv B_{14 *}\left(\sigma_{c} / 0.1\right)^{1 / 2} \times 10^{14} \mathrm{G}$.

Parameter $q$ in eq.(23) is given by $q \approx 0.4\left(B_{14 *}\right)^{-2}$. Figure 1 displays our numerical results for the three models listed in Table 1 for various multipole $l$ values. These results indicate that there exist two branches of equilibrium models. This property is common for all magnetic geometries, regardless of whether the component is poloidol or toroidal. One model features a high field strength of the surface dipole, which corresponds to $B_{14 *}>1$ (equivalent to $q \ll 1$ ). The magnetic energy is proportional to $B_{14 *}^{2}$. For the models in this branch, the elastic force is too weak to sustain the magnetic field deviating from $\vec{B}_{(0)}$, and thus the magnetic field is the same as that of a barotropic MHD model.

Equilibrium models in the other branch, corresponding to $B_{14 *}<1(q \gg 1)$, exhibit different properties. The magnetic energy inside the crust increases in Models $\mathrm{P}$ and $\mathrm{M}$ and approaches a constant in Model T with decrease in $B_{14 *}$. A significantly high magnetic energy $\gtrsim 10^{46} \mathrm{erg}$ is supported by the elastic force, even for a surface dipole field strength of $\sim 10^{13} \mathrm{G}$. This result is explained as follows. The maximum strain is located near the surface in Model P. The additional field $\vec{B}_{(s)}$ always exists inside the crust and approaches zero toward the surface. By reducing the strength of the penetrating field $\vec{B}_{(0)}$, the critical condition near the surface is relaxed, so that the amount of magnetic energy associated with $\vec{B}_{(s)}$ stored deep inside the crust increases. 

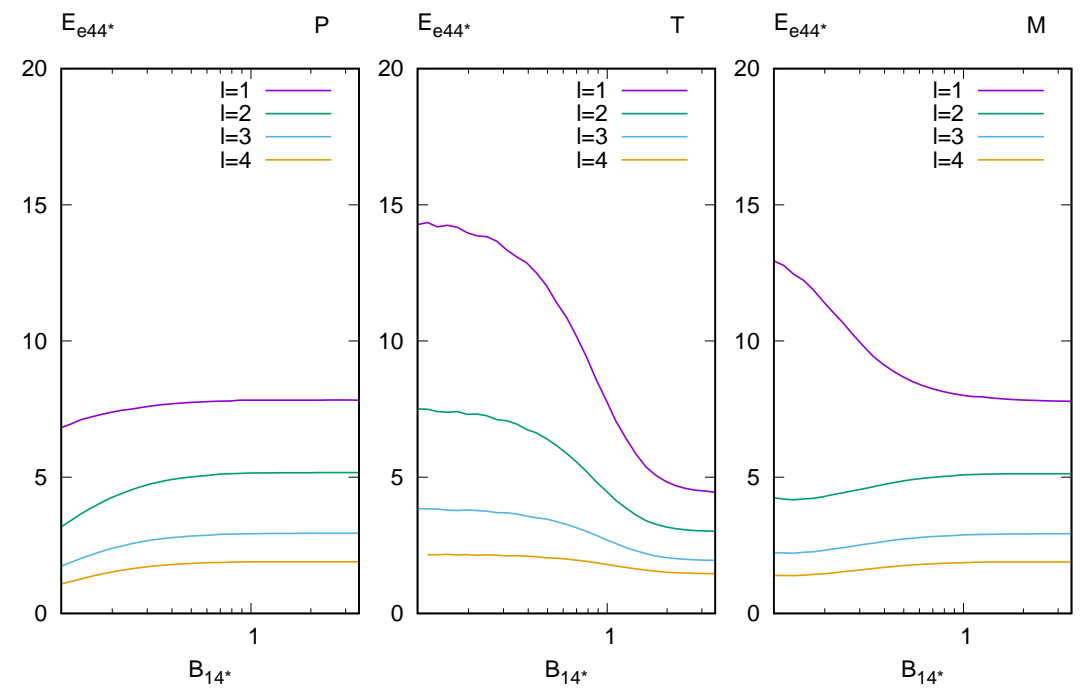

Figure 2. Elastic energy as a function of the surface dipole field strength for the three models listed in Table 1 for various multipole $l$ values.

The maximum strain is located at the middle of the crust in Model T. The dependence of $B_{14 *}$ on the magnetic energy is less sensitive.

The boundary of the two classes is approximately $B_{14 *}=0.3$, which corresponds to a physical value $B_{0}=3 \times 10^{13}\left(\sigma_{c} / 0.1\right)^{1 / 2} \mathrm{G}$. The critical value is presented by $q \sim 4$ in eq. (23). Two distinct classes are demonstrated in the constant $\mu$ models (Kojima et al. 2021), and the results are consistent with those of previous studies for suitable choices of $q$.

Extrapolating our models to either small or large values of $B_{14 *}$ results in further increase in the magnetic energy $E_{\mathrm{mag}}$ in the crust. The energy is several times $10^{47} \mathrm{erg}$, and the field strength correspondingly exceeds $10^{15} \mathrm{G}$. In such an equilibrium model, the magnetic force becomes comparable to the dominant forces near the surface, and our perturbation approach becomes inadequate. These results are applicable to a range of $E_{\mathrm{mag}} \leq E_{c}$, where $E_{c}$ is a few times $10^{47} \mathrm{erg}$.

Figure 2 shows the elastic energy $E_{\text {els }}$ by $E_{\mathrm{e} 44 *}$, where

$E_{\mathrm{els}} \equiv E_{\mathrm{e} 44 *}\left(\sigma_{c} / 0.1\right)^{2} \times 10^{44} \mathrm{erg}$.

The elastic energy changes by a factor of 3 , even for varying $q$ in the range of $10^{-2.5} \leq q \leq 10^{1.5}$. The elastic energy is approximately constant, because all equilibrium models correspond to the maximum shear-strain, where the strength is fixed by the von Mises criterion (22). However, the spatial distribution of $\sigma_{i j}$ changes slightly owing to the combined magnetic fields. The dependence on $l$, i.e., a decrease in energy with $l$ in Fig. 2, mainly originates from the volume integration with the node number $l$, whereas the maximum is fixed irrespective of $l$.

The elastic energy is $E_{\text {els }} \sim 10^{44}-10^{45}$ erg for all models with $\sigma_{c}=0.1$. The magnetic energy $E_{\text {mag }} \sim 10^{45}-10^{47} \mathrm{erg}$ in Fig. 1 always exceeds the elastic energy $E_{\text {els }}$. As discussed previously (Kojima et al. 2021), this fact can be attributed to a large amount of magnetic energy associated with the irrotational part of the magnetic field, which is balanced by the gravity and pressure. Thus, for the equilibrium models, the minor solenoidal component may be balanced by a weak elastic force. Therefore, a magnetic energy larger than the elastic energy is allowed in these models.

\subsection{Magnetic field and shear stress}

Figure 3 displays the magnetic field for the three models listed in Table 1 with $l=2$ and $q=10$, which corresponds to $B_{14 *} \approx 0.2$. The crustal thickness in the figure is enlarged by five times for suitable display. The maximum of the field strength is $B_{p} / B_{0} \sim 31$ in Model P (left panel); $B_{p} / B_{0} \sim 22, B_{\varphi} / B_{0} \sim 114$ in Model T (middle panel); and $B_{p} / B_{0} \sim 28, B_{\varphi} / B_{0} \sim 61$ in Model M (right panel). These fields change considerably from the purely dipolar field $\vec{B}_{0}$, whose magnetic function is the same as that of the middle panel. The poloidal field is confined in Model P, and the strong toroidal field is confined in Models $\mathrm{T}$ and $\mathrm{M}$. Model M may be regarded as a combination of Models $\mathrm{P}$ and $\mathrm{T}$.

Elastic displacements are induced in these models, and the magnitude of shear $\sigma^{i j} \sigma_{i j} /\left(2 \sigma_{c}^{2}\right)$ is displayed in the bottom panels of Fig. 3. The poloidal component $\vec{\xi}_{p}$ is only induced in Model P, and the dominant shear component is found to be 


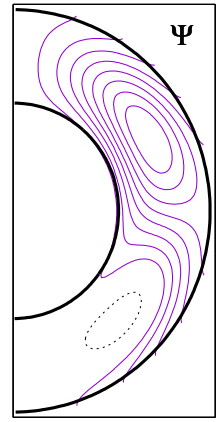

$\mathrm{P}$

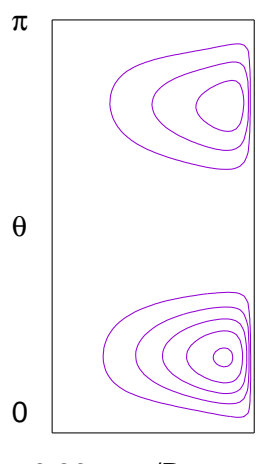

0.90

$r / R$

1.0

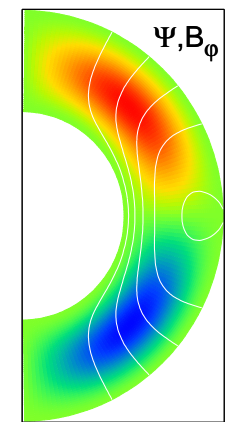

$\mathrm{T}$

$\pi$

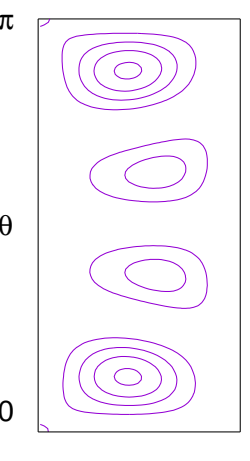

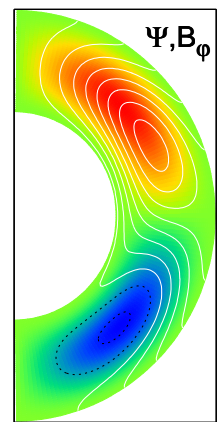

M

$\pi$

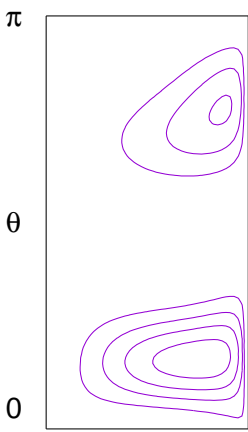

$\begin{array}{llll}1.0 & 0.90 & \mathrm{r} / \mathrm{R} \quad 1.0\end{array}$

Figure 3. Magnetic field (top panels) and magnitude of shear stress $\sigma^{i j} \sigma_{i j} /\left(2 \sigma_{c}^{2}\right)$ (bottom panels) for Models P, T, and M listed in Table 1 , from left to right. The contour of $\Psi$ in the top panels is plotted With an interval of $\Delta \Psi /\left(B_{0} R^{2}\right)=0.1$ in spherical coordinates "enlarged" in the radial direction for display purposes. The dotted lines represent the negative value of the contour level. The color contour represents the toroidal field $B_{\varphi}$ normalized by the maximum value. The contour of $\sigma^{i j} \sigma_{i j} /\left(2 \sigma_{c}^{2}\right)$ in the $r-\theta$ plane is plotted with $\Delta=0.15$ for the crustal region.

$\sigma_{r \theta}=r\left(r^{-1} \xi_{\theta}\right)_{, r}+r^{-1}\left(\xi_{r}\right)_{, \theta}$. Two peaks originate from the additional magnetic field in which the angular function is specified by $l=2$, and the radial function has no node. The position of the maximum shear-strain is not at the center of the crust but is shifted near the surface $r / R \sim 0.99$.

In models with the toroidal component, the axial component $\xi_{\varphi}$ is also induced. The shear component $\sigma_{r \varphi}=r\left(\xi_{\varphi} / r\right){ }_{, r} \propto S$ is dominant for small amplitudes $\left|N_{2}\right|$ in eq.(21), whereas $\sigma_{r \theta}$, which originates from the Lorentz force $j_{p} \times B_{\varphi} \propto S^{2}$, is dominant for large amplitudes. The angular dependence of $l=4$ in Model T shown in Fig. 3 is explained by the dominant component $\sigma_{r \theta}$, although the additional magnetic field is $l=2$. The position of the maximum shear-strain is the geometrical center of the crust. The distribution of the shear magnitude is more similar to that in Model $\mathrm{P}$, whereas the magnetic field in Model $\mathrm{M}$ is a combination of those in Models $\mathrm{P}$ and $\mathrm{T}$, displayed in top panels.

The equilibrium models are critical, because they exhibit the maximum strain $\sigma_{c}$. Energy is released after the crustal fracture when the strain exceeds a threshold. For example, we estimate the strain for Model M. As displayed in the right panel of Fig. 3, the magnitude of the shear stress peaks near $r / R=0.99$. The location corresponds to $\rho \approx 10^{12} \mathrm{~g} \mathrm{~cm}^{-3}$, since the outer boundary $R$ corresponds to the neutron-drip density $\rho_{1}=4 \times 10^{11} \mathrm{~g} \mathrm{~cm}^{-3}$. Assuming a fraction of $10^{-2}$ of the crust near the surface breaks and that $10^{-2}$ of the total magnetic energy is released, the energy is estimated as $\sim 2 \times 10^{44}$ erg. If the energy is supplied from the same fraction of elastic energy, the energy reduces to $4 \times 10^{42} \mathrm{erg}$. These energies are sufficient to induce the violent phenomena observed in the magnetized neutron stars, although there is uncertainty concerning whether the elastic or magnetic energy is the actual source and what fraction of the total energy is released during explosions. The energy estimate also depends on the magnetic field configuration, which introduces another ambiguity.

Beyond the elastic limit, the crust may respond plastically instead of with cracking. It is also important to evaluate to what region the magnetic field is rearranged, as the subsequent magnetic field evolution with plastic flow is significantly different depending on the global or local nature of the flow (Gourgouliatos \& Lander 2021). Understanding the crustal dynamics beyond the elastic limit is critical for further discussions. 

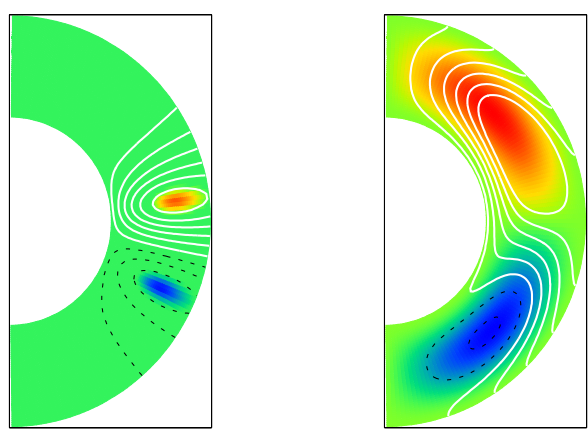

$\mathrm{B}_{\varphi}$

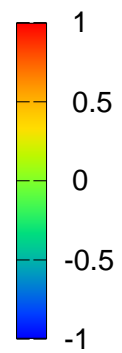

Figure 4. Magnetic field for barotropic MHD model (left) and elastic model (right) for the crust enlarged five times. Dotted lines represent the negative value of the contour level. Color contour represents the toroidal component normalized by the maximum value.

\begin{tabular}{ccc}
\hline Model & $\left(B_{\varphi}\right)_{\max } /\left(B_{\mathrm{p}}\right)_{\max }$ & $E_{\varphi} /\left(E_{\mathrm{p}}+E_{\varphi}\right)$ \\
\hline Barotropic MHD & $9.6 \times 10^{-2}$ & $6.5 \times 10^{-3}$ \\
Elastic Model & 2.0 & 0.84 \\
\hline
\end{tabular}

Table 2. Comparison of models

\subsection{Model comparison}

We consider the effect of the elasticity on the equilibrium models with mixed poloidal-toroidal magnetic fields. In the barotoropic MHD approximation, the current function $S$ is a function of $\Psi$ as discussed in eq.(18), and is selected as follows:

$$
\begin{aligned}
& S=S_{0} \Psi\left(\Psi / \Psi_{\max }-1\right) \Theta\left(\Psi / \Psi_{\max }-1\right) \text { for } \Psi \geq 0 \\
& S=S_{0} \Psi\left(\Psi / \Psi_{\min }-1\right) \Theta\left(\Psi / \Psi_{\min }-1\right) \text { for } \Psi<0,
\end{aligned}
$$

where $\Theta$ is the usual Heaviside step function, and $S_{0}$ is a constant. A similar choice has been performed in previous studies, (e.g., Yoshida et al. 2006; Ciolfi et al. 2009) and the numerical method to calculate the equilibrium model is discussed. We assume that $\Psi_{\min }<0$ and $\Psi_{\max }>0$. The toroidal magnetic field is allowed in two spatially detached regions: $B_{\varphi} \leq 0$ for $\Psi \leq \Psi_{\min }$ and $B_{\varphi} \geq 0$ for $\Psi \geq \Psi_{\max }$, while $B_{\varphi}=0$ for the intermediate. The toroidal field is described by $l=2$ and higher multipoles. The magnetic function $\Psi$ for $\Psi_{\min } \leq \Psi \leq \Psi_{\max }$ is smoothly connected to the exterior field, which is described by $l=1,2$ and higher multipoles.

As discussed in Section 1, obtaining numerical models with large toroidal magnetic-energy is difficult. The model displayed in the left panel of Fig. 4 corresponds to the maximum of the toroidal energy. The equilibrium model with the elastic force is presented in the right panel of Fig. 4 , which is Model M with $l=2$, and the ratio of the field $\operatorname{strengths}$ is $\left(B_{\varphi}\right)_{\max } /\left(B_{\mathrm{p}}\right)_{\max }=2$. In the barotropic model, the toroidal field is spatially localized inside a tiny loop in a meridian plane. In contrast to the barotoropic MHD model, the toroidal field extends over the crust in the magneto-elastic equilibrium model. Thus, a large amount of toroidal magnetic energy is stored.

The comparison between the two models is also summarized via the maximum ratio of the toroidal to poloidal field strengths and energy in Table 2. Elasticity plays a crucial role in sustaining the toroidal magnetic field.

\section{CONCLUDING REMARKS}

In this study, we investigated the equilibrium state of a magnetized neutron star crust, where the shear modulus is proportional to the density. We focused on the magnetic field hidden in the crust, with a field strength that is considerably higher than that of the surface dipole field. The magnetic field geometry containing strong poloidal and toroidal components is not obtained numerically by a barotropic MHD approximation, although it is conjectured to explain observational phenomena occurring in neutron stars. Assuming that the irrotational part of the Lorentz force is balanced by the pressure and gravity, possible equilibrium models with strong fields confined by the elastic force were demonstrated. The solenoidal part of the Lorentz force is typically smaller than the irrotational part, and therefore the weak elastic force is sufficient for the static model. The magnetic field configuration is less constrained, and a large amount of magnetic energy up to $\sim 10^{47}$ erg is sustained by the elastic force. For example, the average field strength $B$ in the crust is $B \sim 3 \times 10^{14} \mathrm{G}$ for $E_{\text {mag }}=10^{46}$ erg, and a strong field ( $>10^{14}$ G) may be hidden even when the surface dipole is low $<10^{13} \mathrm{G}$. This case corresponds to low-field magnetars. However, the elastic force is less effective for a typical magnetar with a surface field strength of $\sim 10^{14} \mathrm{G}$. A small deformation is allowed within a critical limit, and the magnetic field geometry in equilibrium does not deviate from that without the elastic force. 
When the magnetic energy exceeds several times $10^{47} \mathrm{erg}$, the magnetic force of $B \sim 10^{15}$ G becomes comparable to the magnitudes of the pressure and gravity at the surface. The maximum energy stored in the crust depends considerably on the magnetic field geometry; a larger energy is stored when the field is concentrated toward the core because other forces also increase. Magnetic energy in the range of $2.5 \times 10^{45}-4 \times 10^{48} \mathrm{erg}$ stored in the crust with the low surface field of $\sim 10^{11} \mathrm{G}$ (average field strength of $2 \times 10^{14}-5 \times 10^{15} \mathrm{G}$ ) has been considered as a model for the CCO power (Gourgouliatos et al. 2020; Igoshev et al. 2021). However, the large parameters adopted may not provide dynamically stable confinement, although our result for an axisymmetric model does not apply to the 3D magnetic fields considered previously.

The critical structure at the elastic limit is calculated by using a realistic shear modulus. Correct identification of the crustfracture location is crucial for the secular time evolution of the crustal magnetic field. Elastic deformation is induced during the evolution, and evolution beyond a threshold is discontinuous. Such a transition may appear as a burst in a magnetar. The magnetic field at the critical limit is rearranged to model the burst in numerical simulations (Pons \& Perna 2011; Dehman et al. 2020), where the magnetic stress is used to estimate the critical state. Our results show that the maximum shear-strain occurs near the surface ${ }^{1}$. The thickness of the skin layer is $\sim 10^{2} \mathrm{~m}$. The magnetic energy stored there is $\sim 10^{44}$ erg, and the elastic energy is $\sim 10^{42} \mathrm{erg}$. Thus, a difference of two orders of magnitude exists between two energy scales, and it is unclear which energy scale is more relevant to the observed bursts or flares. Partial energy of this order is released during abrupt events, but more precise investigations are required to clarify this.

The estimates strongly depend on the field geometry. The model considered in this study is simple, and the magnetic field is described by a lower multipole extended to the entire crust. Small energy scales, such as $<10^{41}$ erg, in short bursts (Turolla et al. 2015) are related to locally irregular fields, which may be concentrated elsewhere in the interior. Irrespective of the geometry, the effect of the elasticity considered in this study plays a crucial role in magnetic field confinement of $\sim 10^{14} \mathrm{G}$ in the solid crust.

\section{ACKNOWLEDGEMENTS}

This study was supported by JSPS KAKENHI Grant Number JP17H06361, JP19K03850(YK), JP18H01246, JP19K14712, JP21H01078(SK), JP20H04728(KF).

\section{DATA AVAILABILITY}

The data underlying this article will be shared on reasonable request to the corresponding author.

\section{REFERENCES}

Akgün T., Reisenegger A., Mastrano A., Marchant P., 2013, MNRAS, 433, 2445

Archibald R. F., Kaspi V. M., Tendulkar S. P., Scholz P., 2016, ApJ, 829, L21

Armaza C., Reisenegger A., Valdivia J. A., 2015, ApJ, 802, 121

Baiko D. A., Chugunov A. I., 2018, MNRAS, 480, 5511

Bera P., Jones D. I., Andersson N., 2020, MNRAS, 499, 2636

Braithwaite J., 2009, MNRAS, 397, 763

Braithwaite J., Nordlund A., 2006, A\&A, 450, 1077

Braithwaite J., Spruit H. C., 2004, Nature, 431, 819

Caplan M. E., Schneider A. S., Horowitz C. J., 2018, Phys. Rev. Lett., 121, 132701

Chamel N., Haensel P., 2008, Living Reviews in Relativity, 11, 10

Ciolfi R., Ferrari V., Gualtieri L., Pons J. A., 2009, MNRAS, 397, 913

Colaiuda A., Ferrari V., Gualtieri L., Pons J. A., 2008, MNRAS, 385, 2080

De Grandis D., Taverna R., Turolla R., Gnarini A., Popov S. B., Zane S., Wood T. S., 2021, ApJ, 914, 118

De Luca A., 2017, J. Phys., 932, 012006

Dehman C., Viganò D., Rea N., Pons J. A., Perna R., Garcia-Garcia A., 2020, ApJ, 902, L32

Duez V., Mathis S., 2010, A\&A, 517, A58

Duez V., Braithwaite J., Mathis S., 2010, ApJ, 724, L34

Enoto T., Kisaka S., Shibata S., 2019, Reports on Progress in Physics, 82, 106901

Fujisawa K., Eriguchi Y., 2013, MNRAS, 432, 1245

Fujisawa K., Kisaka S., 2014, MNRAS, 445, 2777

Glampedakis K., Andersson N., Lander S. K., 2012, MNRAS, 420, 1263

1 The outer boundary of our model is the interface of the inner and outer crusts. When the toroidal magnetic field is extended to the outer crust, the region becomes susceptible to cracking because of the lower shear modulus. The total magnetic energy sustained by the elastic force is therefore reduced. Conversely, when the field is confined in deep interior regions, the magnetic energy increases. These features can be discussed in detail only after the magnetic field configuration is clarified. 
Gotthelf E. V., Halpern J. P., Alford J., 2013, ApJ, 765, 58

Gourgouliatos K. N., Lander S. K., 2021, MNRAS, 506, 3578

Gourgouliatos K. N., Cumming A., Reisenegger A., Armaza C., Lyutikov M., Valdivia J. A., 2013, MNRAS, 434, 2480

Gourgouliatos K. N., Hollerbach R., Igoshev A. P., 2020, MNRAS, 495, 1692

Horowitz C. J., Kadau K., 2009, Phys. Rev. Lett., 102, 191102

Igoshev A. P., Gourgouliatos K. N., Hollerbach R., Wood T. S., 2021, ApJ, 909, 101

Kaspi V. M., Beloborodov A. M., 2017, ARA\&A, 55, 261

Kojima Y., Suzuki K., 2020, MNRAS, 494, 3790

Kojima Y., Kisaka S., Fujisawa K., 2021, MNRAS, 506, 3936

Lander S. K., Gourgouliatos K. N., 2019, MNRAS, 486, 4130

Lander S. K., Jones D. I., 2009, MNRAS, 395, 2162

Lander S. K., Jones D. I., 2011a, MNRAS, 412, 1394

Lander S. K., Jones D. I., 2011b, MNRAS, 412, 1730

Lander S. K., Jones D. I., 2012, MNRAS, 424, 482

Malvern L. E., 1969, Introduction to the mechanics of a continuous medium. Englewood Cliffs, N.J. : Prentice-Hall

Markey P., Tayler R. J., 1973, MNRAS, 163, 77

McLaughlin M. A., et al., 2003, ApJ, 591, L135

Mitchell J. P., Braithwaite J., Reisenegger A., Spruit H., Valdivia J. A., Langer N., 2015, MNRAS, 447, 1213

Pons J. A., Perna R., 2011, ApJ, 741, 123

Rea N., et al., 2012, ApJ, 754, 27

Rea N., et al., 2013, ApJ, 770, 65

Rea N., Viganò D., Israel G. L., Pons J. A., Torres D. F., 2014, ApJ, 781, L17

Reisenegger A., 2009, A\&A, 499, 557

Sur A., Haskell B., Kuhn E., 2020, MNRAS, 495, 1360

Tayler R. J., 1973, MNRAS, 161, 365

Tayler R. J., 1980, MNRAS, 191, 151

Tomimura Y., Eriguchi Y., 2005, MNRAS, 359, 1117

Turolla R., Zane S., Watts A. L., 2015, Reports on Progress in Physics, 78, 116901

Uryū K., Yoshida S., Gourgoulhon E., Markakis C., Fujisawa K., Tsokaros A., Taniguchi K., Eriguchi Y., 2019, Phys. Rev. D, 100,123019 Ushomirsky G., Cutler C., Bildsten L., 2000, MNRAS, 319, 902

Wright G. A. E., 1973, MNRAS, 162, 339

Yoshida S., 2019, Phys. Rev. D, 99, 084034

Yoshida S., Eriguchi Y., 2006, ApJS, 164, 156

Yoshida S., Yoshida S., Eriguchi Y., 2006, ApJ, 651, 462 